\title{
GENOMICS
}

\section{Whole genome sequencing has gone to the dogs}

Plassais, J. et al. Nat. Commun. 10, 1489 (2019)

In man, the difference in height between someone who is 5' 5 " and someone who is 6 ' 5 ' is influenced by hundreds of different genes. In man's best friend, the variation in size between a tiny Chihuahua and a gigantic Great Dane can be explained by as few as just 15 to 20 genes, according to a recently completed whole genome sequence analysis of 722 canines sampled from 144 modern breeds, plus 54 wild canids and 100 village dogs. "That's extraordinary," says lead author Elaine Ostrander of the National Institutes of Health National Human Genome Research Institute. "We honestly didn't know that it would be that few."

It makes sense though, she says, when you consider the evolutionary timescales involved. Humans have been evolving for millions of years while dogs, Canis familiaris, only split from grey wolves on the road to domestication on the order of tens of thousands of years ago. "That's an evolutionary drop in the bucket," she says. Even more striking, most modern breeds have only been in existence since Victorian times. In humans, that means diversity is influenced by lots of genes that have small effects, Ostrander explains, while the opposite is true in dogs: few genes with big effects account for all the different shapes, sizes and colors observed in canines across the world, as well as less visual phenotypes such as behavior and susceptibility to different diseases.

Companion canines are not the typical lab animal-they are pets, exposed to the vagaries of the world just like their humans and susceptible to ailments such as cancer that can present in people in similar ways. Ostrander has been studying dogs and their genomes since the 1990s. "There is such extraordinary variation in domestic dogs, it's more than we see in any other land mammal," she says. "With the right genomic tools, we would be able to find the genes that control that variation." Breed is an important key, as precisely established breed standards dictate remarkably specific details about how an animal belonging to a particular breed should look and behave. Over the past few years, Ostrander and her collaborators have been collecting DNA samples from dogs all over the world, focusing on show dog lineages with carefully recorded pedigrees that can be used to map relationships between different animals. The response has

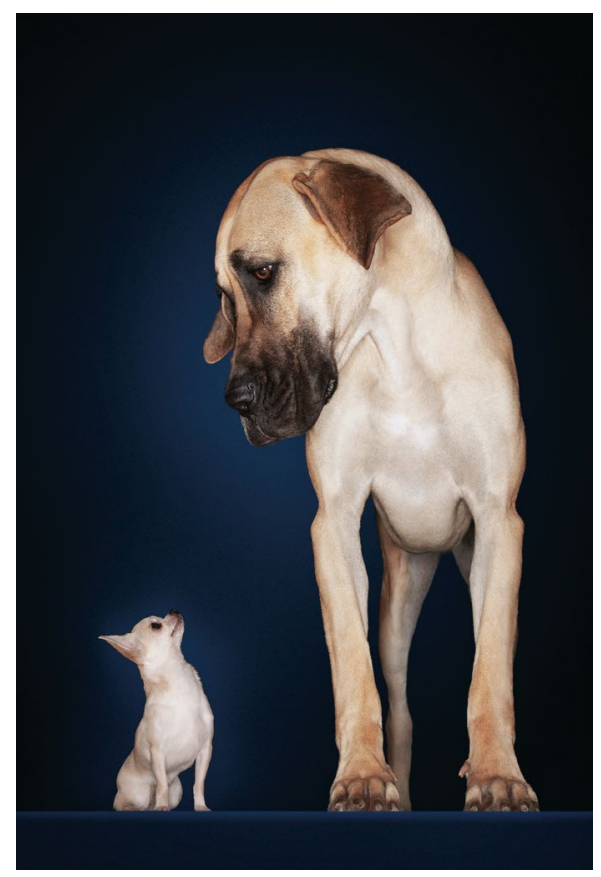

Little dog, big dog-it's all in the genes. Credit: moodboard/Cultura/Getty

been overwhelming, she says. "We virtually never get turned down." Her freezer contains over 34,500 samples, and counting.

The 'dog' genome was first sequenced in 2005-the particular animal chosen was a purebred female boxer named Tasha. Genomics has come a long way since. In the past, state-of-the art technologies could indicate regions of interest for a particular mutation that's likely responsible for a certain phenotype in a given breed. The problem? Those regions could be millions of base pairs long; finding a meaningful mutation and not one simply the result of genetic drift could be a challenge, to say the least. But whole genome sequencing - identifying every single base pair in a sample-has gotten cheaper and computers' ability to analyze and compare different genomes more powerful. But first, Ostrander and her colleagues needed to determine whether a genome-wide association study could be completed with whole-genome sequence data. Comparing morphology against breed standards was, she says, "the cleanest way to do proof-of-principle, to say, 'does this work or not?"' It did.
Thanks to the meticulousness of $\operatorname{dog}$ breeders, the research team didn't need to record and compile measurement data for every single animal that was sampled-they only needed to compare against the standard to look for genes responsible for phenotypic details such as leg length, body weight, or ear shape. From a data set consisting of 91 million genetic variants, they analyzed 16 morphological phenotypes in all and narrowed in on a number of genes that are likely responsible for the different features. It revealed much about dogs themselves, but translational trends are also apparent. Take the big dogs: the larger the dog, the shorter its lifespan tends to be. The analysis indicated a handful of genes responsible for size in dogs. In humans, when such genes are mutated, condition such as obesity and metabolic disease often follow, says Ostrander.

The initial analysis was published in Nature Communications, while the dataset itself is freely available online through the National Center for Biotechnology Information. This effort is also part of a much larger one, the Dog $10 \mathrm{~K}$ sequencing project, an international collaboration Ostrander helped initiate in 2015 to amass 10,000 canine whole genome sequences for reference and analysis.

There is still much to come-dozens of other traits were mapped but not included in the current publication, Ostrander says. Continuing on with canines, her lab is interested in exploring behavior as well as the origins of domestic dogs next; a few members have eyes on disease traits too. Moving beyond breed standards does become trickier and requires more follow up with the actual animals. To look at the underpinnings of different diseases, for example, they've asked participants to keep them up-to-date on the health status of the dogs sampled. If a dog goes on to develop a disease, the researchers can then compare that animal's genome against others who have remained healthy. Owners aren't shy"they give us more information than you could ever want," says Ostrander.

All for the good of the dogs themselves, and maybe one day for their people, too.

Ellen P. Neff

Published online: 6 May 2019

https://doi.org/10.1038/s41684-019-0315-9 